\title{
A New Optical Realization of the ICRS
}

\author{
George A. Gontcharov, Anna A. Andronova, Edward V. Kornilov \\ Pulkovo observatory, 196140, Saint-Petersburg, Russia
}

Oleg A. Titov

Astronomical institute of Saint-Petersburg University, Bibliotechnaya 2, Petrodvorets, Saint-Petersburg, 198904, Russia

\begin{abstract}
A new optical realization of the ICRS is built upon the data of the Hipparcos catalog and 60 observational and compiled astrometric ground-based catalogs of the $20^{\text {th }}$ century reduced to the Hipparcos system. It is in print as two catalogs. The 'Proper Motions of Fundamental Stars' catalog (PMFS) contains proper motions of 4638 stars common to the Hipparcos and Basic FK5/FK5 Extension improved to the level of 0.5 mas. 1800 of the stars are the photocenters of stellar systems unresolved in the astrometric observations. The duplicity parameters of those systems were collected in the 'Astrometric Binaries' catalog $(\mathrm{ABC})$. The photocenter nonlinear motions of several hundred of those astrometric binaries were detected and separated from their proper motions. The periods and amplitudes of several astrometric binaries are disscussed in detail. This research will be continued for all Hipparcos stars observed from the ground in the $20^{\text {th }}$ century. The web site is http://www.geocities.com/georgegontcharov/
\end{abstract}

\section{Introduction}

The 'instantaneous' Hipparcos proper motions sometimes differ from 'long-period' ground-based ones because many stars (so called, astrometric binaries) move nonlinearly due to some hidden massive satellites. In these cases one observes the nonlinearly moving photocenters of unresolved stellar systems. Thus, the Hipparcos catalog (hereafter HIP) is not a perfect realization of the ICRS at optical wavelengths. A direct combination of the HIP with ground-based astrometric results can improve the HIP proper motions (Gontcharov \& Kornilov, 1997).

\section{The precise proper motions of $\mathbf{4 6 3 8}$ fundamental stars}

We used the positions, parallaxes and radial velocities from the HIP, proper motions from several ground-based compiled catalogs and positions from 57 observational ground-based catalogs with mean epochs from 1938 to 1994. The observational catalogs were obtained with meridian instruments and astrolabes 
at Beijing, Beograd, Bordeaux, Brorfeld, Calern, Cerro Calan, La Palma, Nikolaev, Perth, Pulkovo, Washington and other observatories. The full list of the catalogs is published elsewhere.

The differences $\Delta \alpha$ and $\Delta \delta$ in the sense of 'observational catalog minus HIP at the observational catalog epoch' were calculated and then approximated by Legendre-Hermite-Fourier functions. The functions, interpreted as the systematic errors of the ground-based catalogs, were removed. The resultant series of star positions for the various epochs referred to the ICRS were used to derive new proper motions independent from the HIP. The mean weighted proper motions from the HIP, GC, N30, FK5, N70E, CMC9 and KSV2 (Time Service Catalog 2) were used for the first of three iterations.

The derived proper motions together with positions, parallaxes and radial velocities from the HIP can be considered as a new optical realization of the ICRS (very close to the HIP for the epoch 1991.25). It is presented by the Proper Motions of Fundamental Stars catalog (PMFS) (Gontcharov et al., 2000a) and the 'Astrometric Binaries' Catalog (ABC) (Gontcharov et al., 2000b) containing parameters of the stellar systems unresolved in space and/or ground-based astrometric observations.

The advantage of the PMFS proper motions over ground-based ones (such as from the FK5) is that the differences 'PMFS minus HIP' do not show any noticeable systematic dependence on the equatorial coordinates. On the other hand, the advantage of the PMFS proper motions over HIP ones is that they reflect the star motions over decades, not only in the course of the 3.36 years of the mission. Namely, the nonlinear motions of 400 stars were directly separated into their proper motions and periodic motions of the photocenters around their barycenters. The proper motions of another 1000 stars were separated from their nonlinear motions by the large differences of the PMFS and HIP proper motions of the stars.

The precision of the components of the proper motion in the PMFS is 0.5-2.0 mas/year depending on possible duplicity of the star, the coordinate ( $\alpha$ or $\delta$ ), and membership in the FK5 System (Basic or Extension, with more or less observations from the ground). The median precision of the PMFS proper motions is $0.5 \mathrm{mas} /$ year for $\alpha$, and $0.7 \mathrm{mas} /$ year for $\delta$.

\section{Some examples of astrometric binaries}

Let's consider several astrometric binaries. As expected, the PMFS proper motions of the stars are closer to the 'long-term' proper motions from the FK5 and FK6 (Wielen et al., 1999) than to the 'instantaneous' ones from the HIP.

Some of the stars are known as visual, spectroscopic or astrometric double or triple systems. But no periodic motion of components or photocenter has ever been observed. The amplitudes and periods of the motion are now estimated astrometrically. Fig. 1 - Fig. 4 show the variations of right ascension and declination with time in mas between the individual catalog positions and the PMFS of selected systems. The mean linear motion is subtracted to show more clearly some nonlinear components of the motion. Every dot with an error bar is the result of one observational ground-based catalog. The HIP position is at 

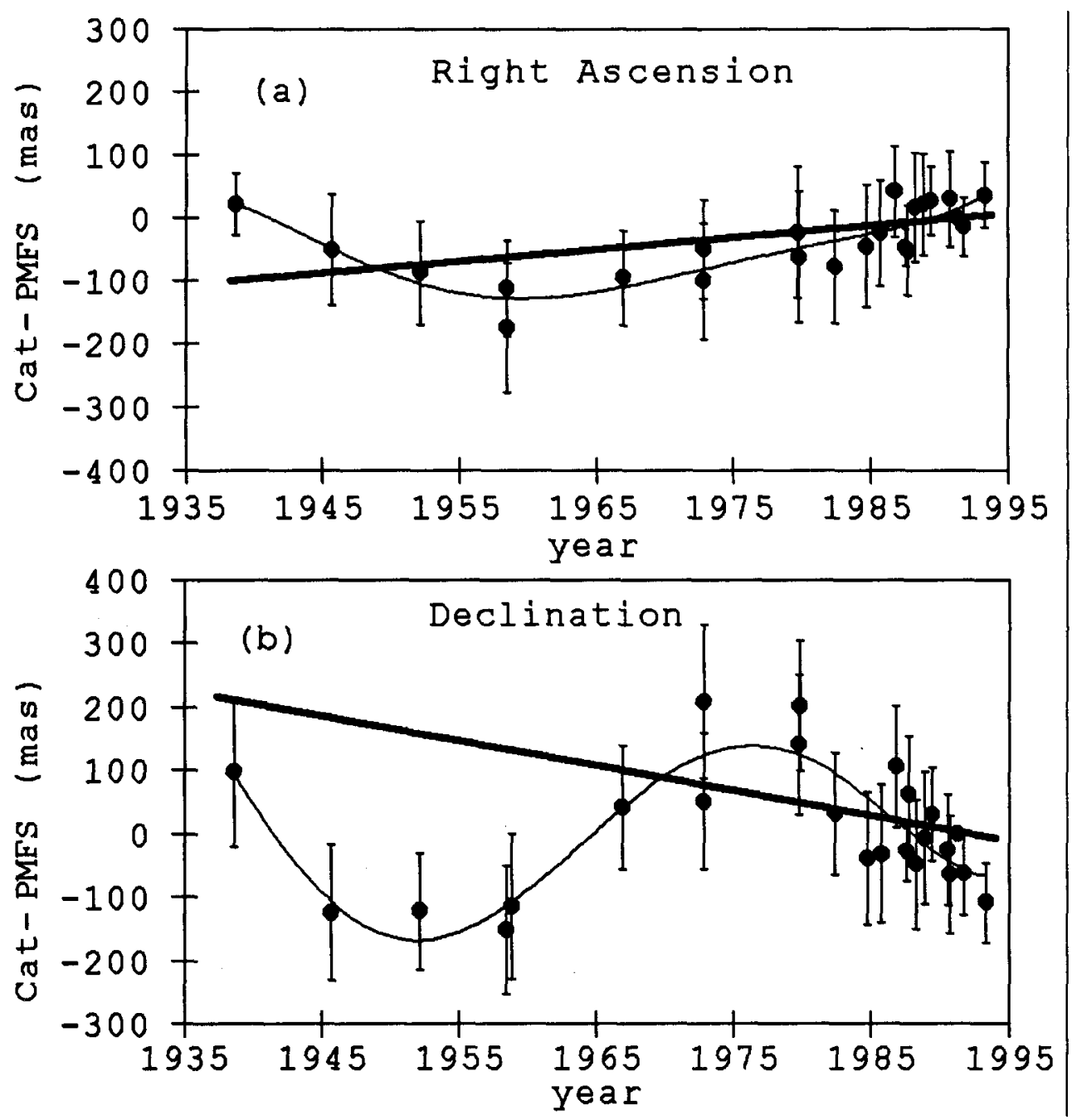

Figure 1. HIP 4427 nonlinear motion: a right ascension, $b$ declination

epoch 1991.25. The thick straight lines reflect the HIP proper motions. The curves are drawn to show the real coordinate variations.

For all the systems there is an interrelation between the variations of $\alpha$ and $\delta$ so that an elliptical orbit can be determined describing the motion. Moreover, all parameters of the systems are consistent with the constraining formula: $\sum M=$ $a^{3} /\left(\pi^{3} \cdot P^{2}\right)$, where $a$ is the semi-major axis in mas, $\pi$ the parallax in mas, $P$ the orbital period in years, and $\sum M$ the sum of the masses of the components in solar mass. The latter, being a function of absolute magnitudes, was roughly evaluated from the HIP data. 

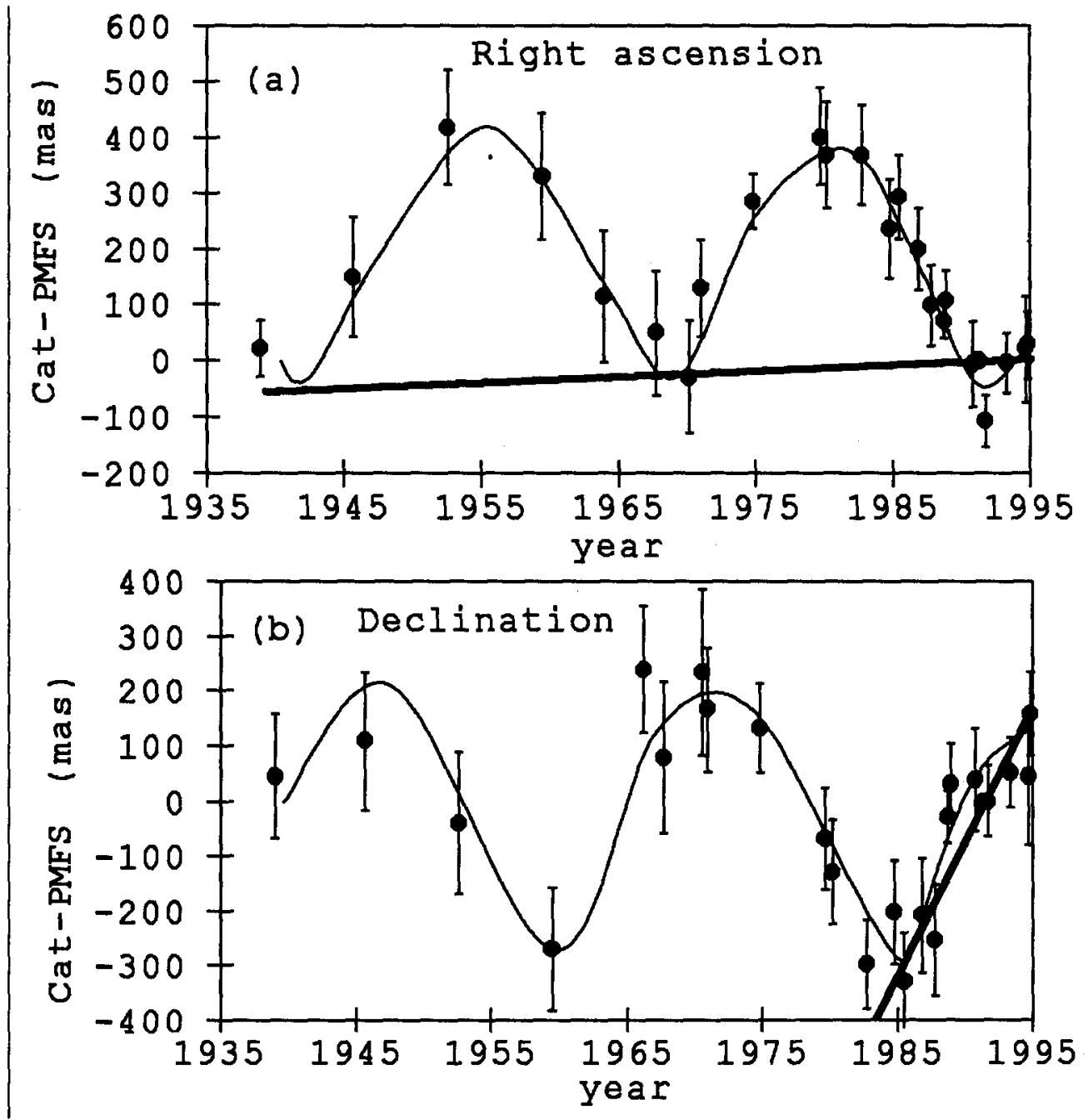

Figure 2. HIP 11072 nonlinear motion: a right ascension, $\mathbf{b}$ declination 

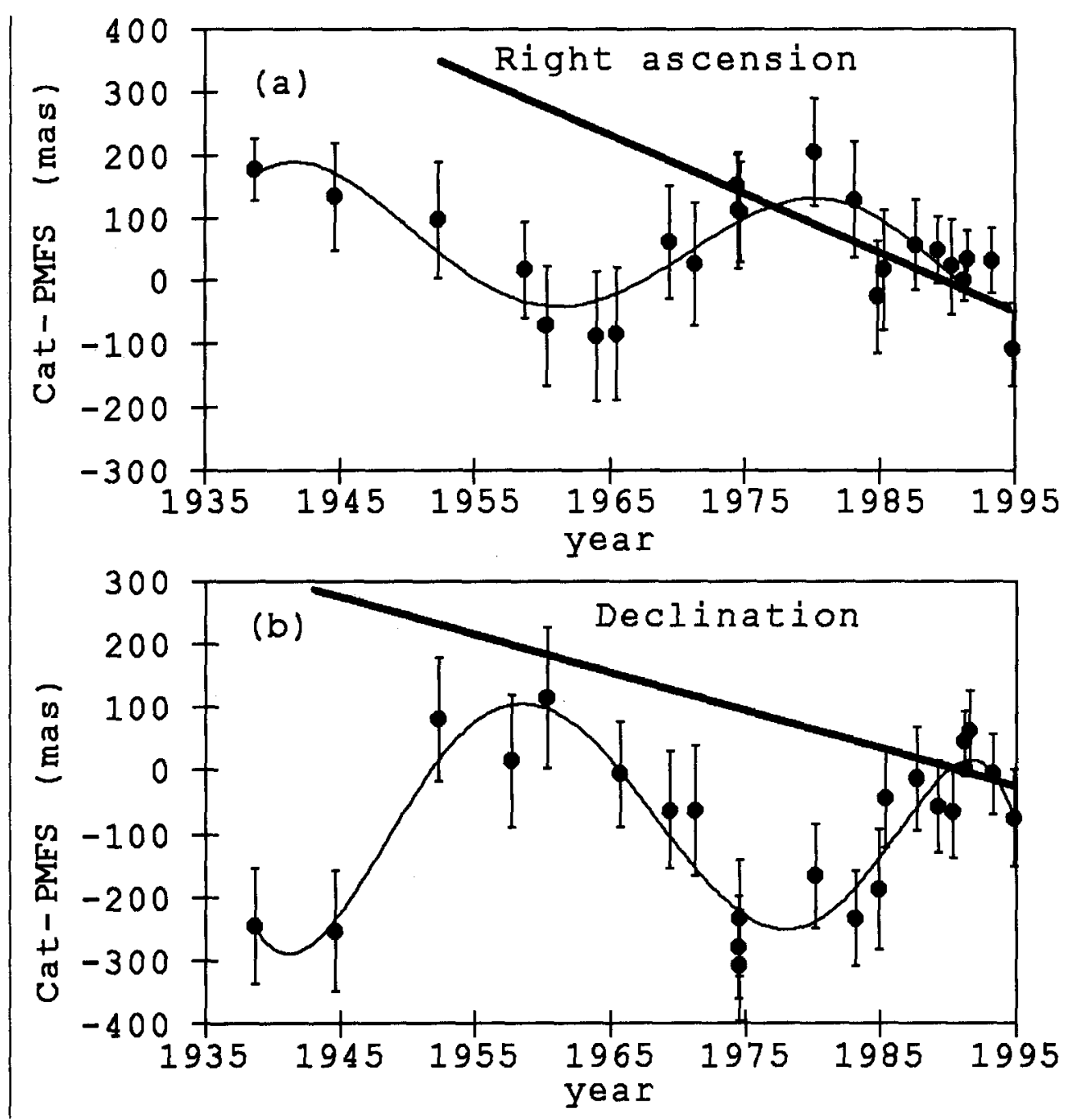

Figure 3. HIP 45699 nonlinear motion: a right ascension, b declination 

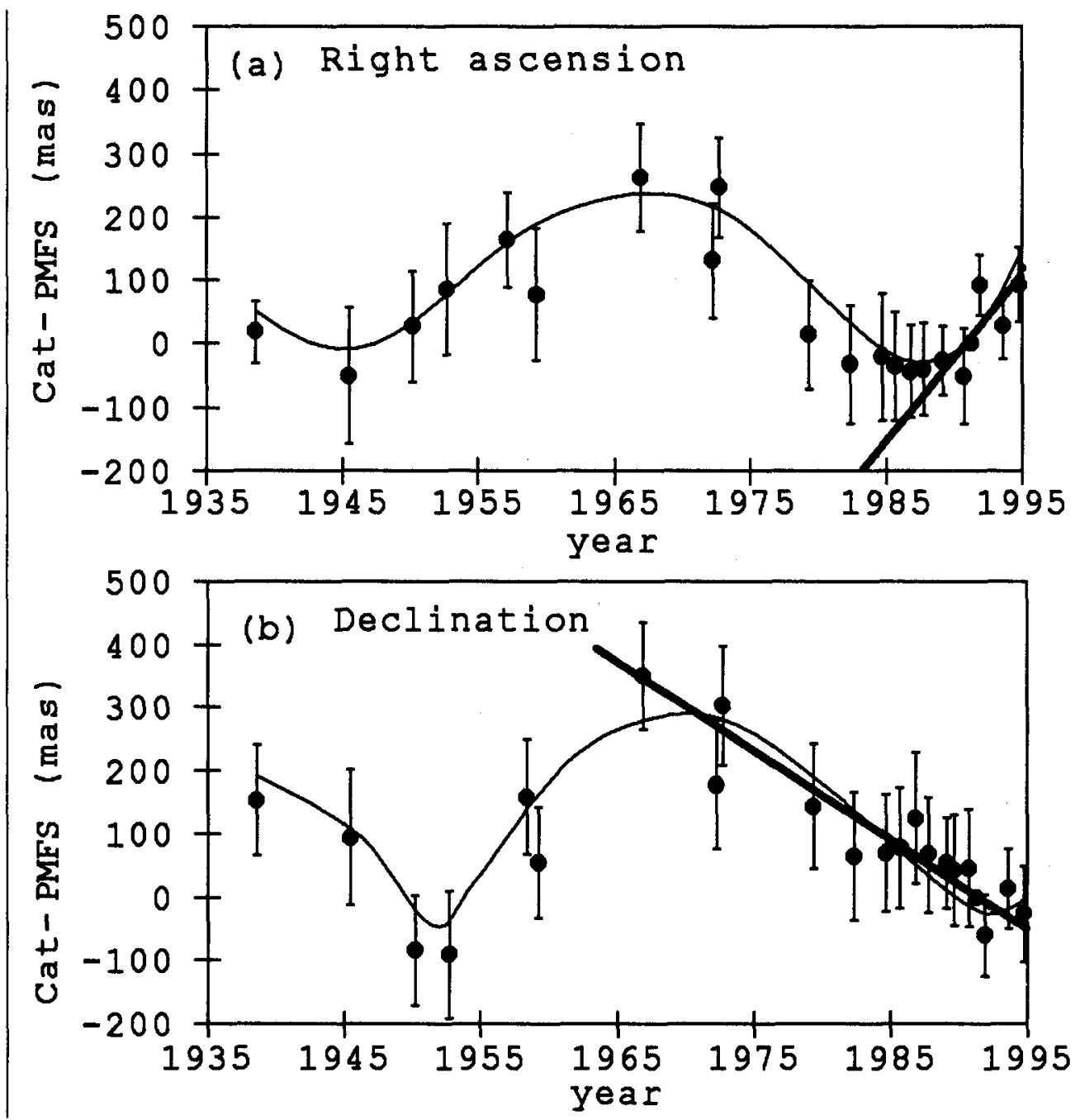

Figure 4. HIP 116727 nonlinear motion: a right ascension, $\mathbf{b}$ declination 


\section{Conclusions}

The majority of the fundamental stars and many HIP stars are members of double and multiple stellar systems. The majority of the stellar components of the systems has not yet been discovered. Therefore,

1. any comparison of astrometric catalogs (such as FK5 and HIP) without separating the stars into the single and non-single ones makes no sense, and

2. any optical realization of the ICRS is far from perfect until all the stellar systems are investigated for hidden star-like bodies and the astrometric parameters of the systems are related to their barycenters,

Acknowledgments. This research was supported by the Russian Foundation for Basic Research (RFBR), project number 97-02-17111.

\section{References}

Gontcharov, G. \& Kornilov, E., 1997, in Journées 1997, systèmes de référence spatio-temporels, J. Vondrak \& N. Capitaine, (eds.) Observatoire de Paris and Astronomical Institute in Prague, 134.

Gontcharov, G. A., Andronova, A. A., Titov, O. A., \& Kornilov, E. V., 2000a, Publ. Pulkovo Obs. (Izvestia glavnoy...), 214 (in press).

Gontcharov, G. A., Andronova, A. A., Titov, O. A., \& Kornilov, E. V., 2000b, Publ. Pulkovo Obs. (Izvestia glavnoy...), 214 (in press).

Wielen, R., Schwan, H., Dettbarn, C., Lenhardt, H., Jahreiß, H., \& Jährling, R., 1999, Veröff. Astron. Rechen-Institut Heidelberg, 35. 